\title{
Sense Cup: A Design of a New Interactive Holistic Sense Convergence Device for Digital Storytelling
}

\author{
Hyun Sang Cho ${ }^{1,2}$, Soohyun $\operatorname{Lim}^{1}$, Tatsuya Yamazaki ${ }^{2}$, and Minsoo Hahn ${ }^{1}$ \\ ${ }^{1}$ Digital Media Laboratory, Information and Communications University, \\ 517-10 Dogok-dong, Gangnam-gu, Seoul 135-854, S. Korea \\ haemosulicu.ac.kr \\ ${ }^{2}$ Universal City Group, Knowledge Creating Communication Research Center, NICT, \\ 3-5 Hikaridai, Seika-cho, Soraku-gun, Kyoto 619-0289, Japan
}

\begin{abstract}
In this paper, we propose "Sense cup" as a new design concept of a device for enhancing the digital storytelling by serving interactive five senses convergence experience. We designed the cup for providing holistic sense experiences with beverage that synchronized with digital contents such as movies and music and catch the user reaction to generate interaction between users and contents provider.
\end{abstract}

Keywords: Sense convergence, Digital storytelling, Immersion, User experience.

\section{Introduction}

Storytelling is one of traditional sharing methods of knowledge and emotion and digital storytelling is a storytelling enhanced by digital technologies in digital media. The term "digital storytelling" has gotten popularity from the first digital storytelling festival that was held in Colorado at 1995 [10]. Joe Lambert, co-founder of the center for digital storytelling [12] said, "Digital storytelling begins with the notion that in the not too distant future, sharing one's story through multiple medium of imagery, text, voice, sound, music, video and animation will be the principal hobby of the world's people".

The main features of the digital storytelling are flexibility, universality, and interactivity [2]. By the flexibility of digital media, traditional linear (sequential) storytelling can be expanded to the nonlinear storytelling such as hypertext storytelling [3]. The universality helps users generate their own stories easily by using cheap and high performance digital technologies. The success story of YouTube shows the impact of the digital storytelling. The interactivity permits users to affect or change the flow of contents (main and ambient) by their decision at every branch of a story. For interactivity, we expanded the concept not only related with the sequence of a story but also related with holistic sense experience that enhances the connectivity between the content of story and users.

Herbert Marshal McLuhan wrote, "The media is an extension of our five senses as we use each of them to communicate to the rest of the world [7]" in his famous book 
"The Medium is the Message". He insisted that the five senses are all of the gates to get information from the outer including the environment and the other human beings. We believed that the holistic sense convergence experience would dramatically enhance the interactivity of digital storytelling. However, the current multimedia technologies are overly inclined to the visual and the auditory information because of the technical feasibility. Recently there have been studies on emphasizing holistic sense convergence as experience to promote effective user communication by giving realistic user experience especially for marketing and there are many efforts to embody other senses [1]. The most successful field of sense except visual and auditory is haptic technology for a sense of touch. There are several researches and commercial haptic display products for giving users kinetic feedback and feeling of texture of a virtual object [9]. However, the sense of smell and sense of taste still has limitations and there are few successful examples of a device, which serves five senses convergence experiences. Two main reasons might be the unbalance among each of sense technology level and pursuit of too broad universal solution.

In this paper, we propose "Sense cup" as a new design concept of a device for enhancing the interactive characteristics of digital storytelling by serving interactive five senses convergence experience. We designed the cup for providing holistic sense experiences with beverage that synchronized with digital contents such as movies and music and catch the user reaction to generate interaction between users and contents provider. In the remaining of this paper, we explain the limitation and solution of five senses convergence and introduce the design concept of Sense cup and the several implementation examples as future work.

\section{Design Concepts of Sense Cup}

\subsection{Sense Convergence}

Sense cup inherited beverage based five senses convergence scenario from our former work "Tea Table Mediator [6]". Fig. 1 (a) shows the convergence relation among five senses. In the former research, senses from tea (or beverage) such as olfactory, taste, haptic, and visual sense are augmented by sense information digital lamp which has visual (LED pattern), audio (sound), and haptic (wind) information display. We shrank the tabletop to a cup.

The most serious problem on holistic sense convergence is providing of a sense of taste and a sense of smell. We will introduce details of our concept of solution at section 2.2. It has five sense display channels (visual, taste, and odor information are provided at a dish on the top of the cup, sound is provided at a bottom-side speaker, and haptic is provided at side of cup) and one physical movement detection channel. The system also designed to detect the movement of cup from a three axes accelometer for deduction of user's status (physical and psychological status such as concentration, boredom, or surprise from inference from the physical movement) which is synchronized with contents. We are going to build a psychological user state model based on hand movement from psychological researches.

One of our sense convergence scenarios is as follow. When a scene of a movie changes to pine tree forest, pine taste beverage with pine odor will be supplied to a 


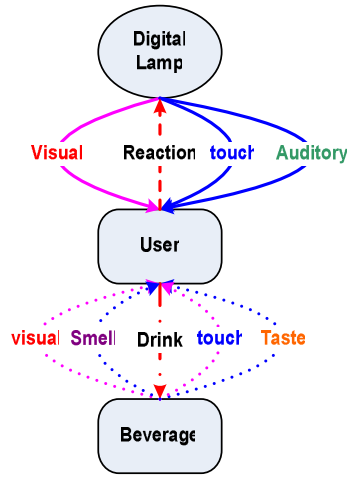

(a) Tea Table Mediator

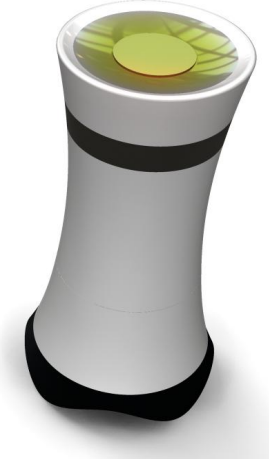

(b) Appearance of Sense cup

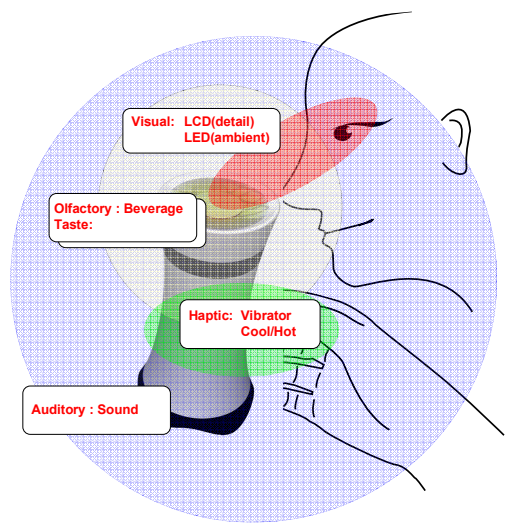

(c) Range of five senses

Fig. 1. Sense convergence of sense cup

dish at the top of a cup. In the bottom of the liquid, the LCD shows the detail images of pine trees and the LEDs show waves of tree according to wind. At the same time, the sound and vibration present the movement of wind in the forest.

A cup is one of everyday objects, which serves beverage, and by drinking beverage on morning or at teatime break people can relax and feel, that they are sharing time with others [5]. There have been several trials to enhance the metaphor of cup by digital technologies [4][5].

\subsection{Displays of Five Senses}

The visual display consists of an LCD and a circular LED array. The LCD shows detail information and LEDs show abstractive shape or movement such as ambient of a scene, the waves, and movement of flowers. The detail display sends clear messages for a case of marketing promotion. The ambient display promotes the curiosity for ludic applications [8]. A bottom-side equipped speaker provides sound information.

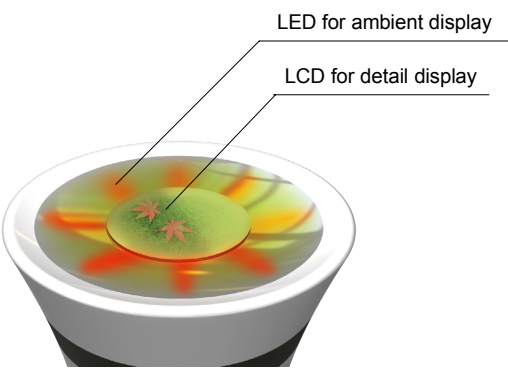

(a) Visual display at top

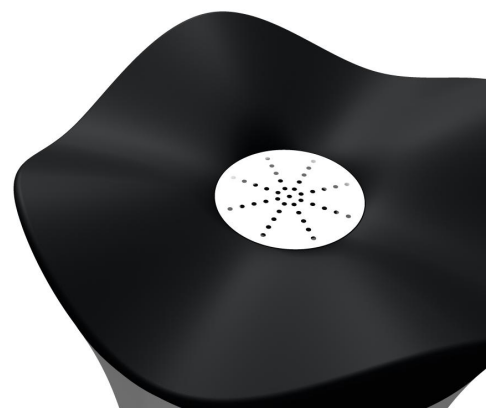

(b) Sound speaker at bottom

Fig. 2. Visual display and audio system 


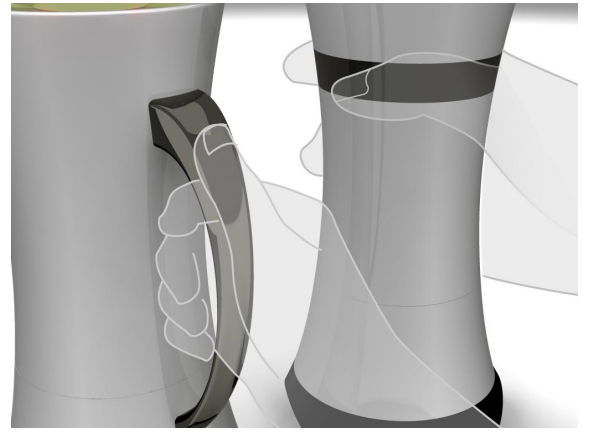

(a) Hand contact with hapic position
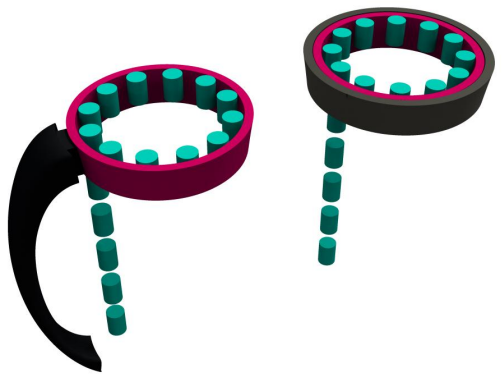

(b) Points of vibrators/hot/cool elements

Fig. 3. Haptic display

The haptic display consists of array of vibrators and electrical hot and cool devices. We assume that a position of hands is almost same for every grab (Fig. 3 (a)) and it is possible to serve tactile information by combining the two kinds of information. The vibrators are equipped vertically and circularly. The circular distribution can be expanded to spiral distribution then, for instance, a user can feel the swirly sink into the bottom of lake or whirlwind of a fairy. Electrically operated cool and hot elements are equipped with a thermal conductor band at the location where directly contacts with hands and provide change of temperature instantly.

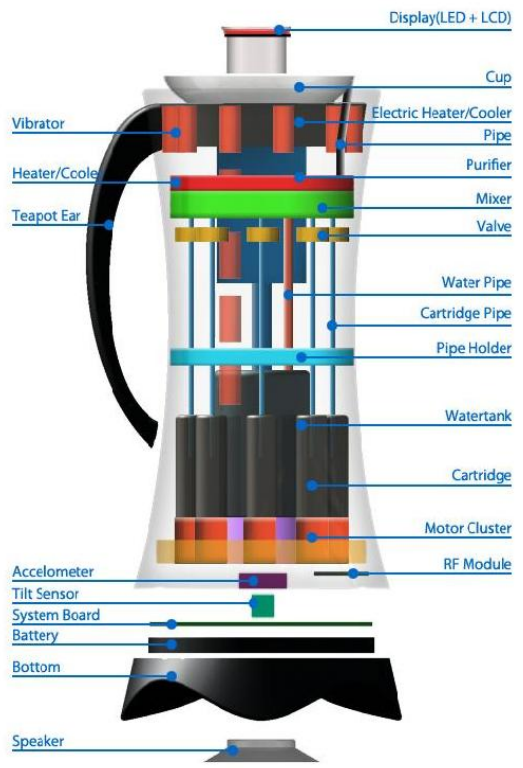

(a) Internal structure of Sense cup

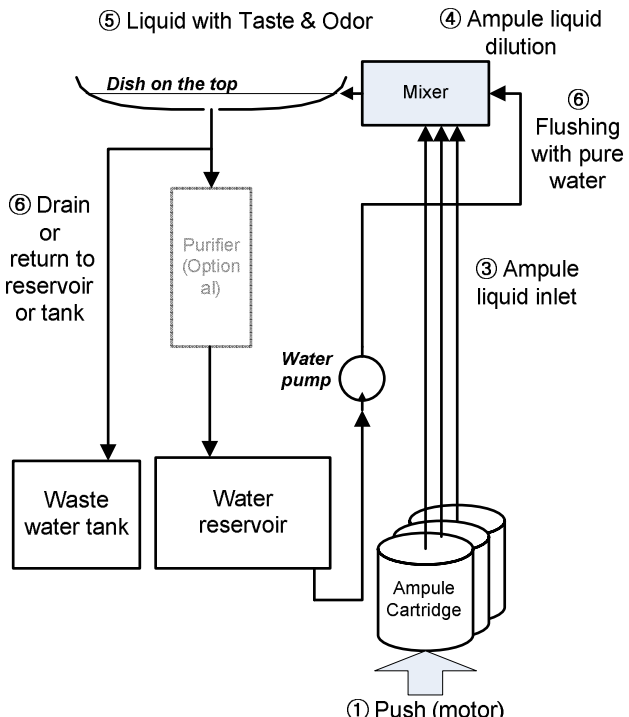

(b) Taste and odor operation

Fig. 4. Taste and odor display 


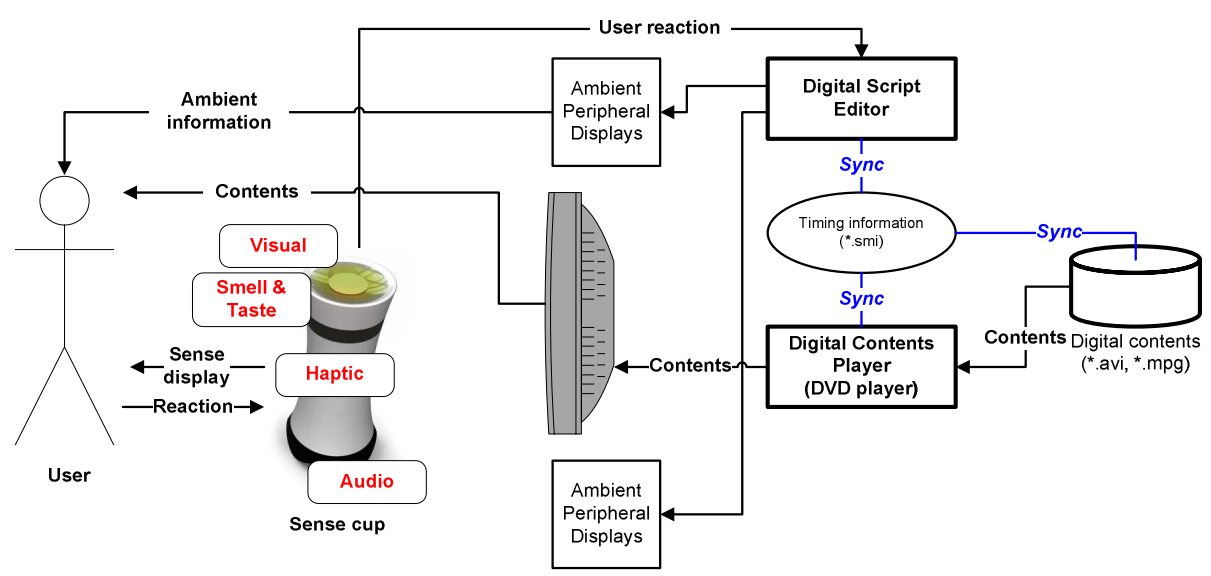

Fig. 5. Configuration of overall Sense cup system

One of the main problems of the sense of taste is a lack of reality of chewy feeling. In earlier stage, a piece of taste-printed paper served a taste. However, it was not successful because of the chewing feeling was mismatched with the taste and the paper was replaced with a wafer [11]. Users could byte and could chew the wafer but it still differed from real feeling. We supposed the solution to overcome the problem by serving a beverage. Since we are familiar with many liquid types of foods such as juice, tea, and alcoholic beverages, it can be possible to solve the mismatch problem. The liquid type food can solve the problem of the sense of smell at same time. The problems of the technologies of odor are; first, there are no definite model and elements of the odor yet, and second, it needs a relatively large volume of air space for serving odors and it is difficult to maintain the intensity and difficult to remove residual odors. In the concept of Sense cup, the odor was generated from the liquid in the dish and the range of the odor is restricted to small space around the dish. A user can smell the odor when he/she wants to drink the liquid or move the cup to near at his/her nose. The odor will be removed quickly as soon as the liquid is drained and flushed. Fig. 4 (b) shows whole process of the taste and odor operation.

\section{Overall System Configuration}

Fig. 5 shows the whole system configuration. It mainly consists of a digital content play system, a digital script editor, and Sense cup and ambient displays. The digital content play system is conventional devices such as a DVD player or a CD player. The digital script editor edits a timing chart of five senses convergence display script. The timing stamp can be obtained from caption files (for example, *.smi or *.srt files) if the content is a moving picture or it can be generated manually. The sense display information is sent to Sense cup by pre-edited timing sequence through wire (RS$232 \mathrm{C} / \mathrm{USB} /$ Ehternet) or wireless (Blutooth/Zigbee/WiFi) communication channels. In current design, the sense information in the script is managed separately from content 
but it can use standardized methods such as Representation of Sensory Effects information (RoSE) of MPEG [13].

As we described in section 2.1, the cup detects movement of user's hands. The movement of hands can show useful information of a user. When a user concentrates on a current content, the movement of a cup is relatively stationary. If a user feels boredom on the content, user will move the cup by various patterns and move the cup abruptly if he/she feels surprise. The editor operates several ambient displays (light/sound/physical movement) for enhancing the user's concentration and interest by the results of the analysis or changes the content as a nonlinear storytelling method.

\section{Conclusion}

In this paper, we showed the conceptual design of Sense cup as a new device of interactive holistic sense experience for digital storytelling. The digital storytelling has been used in various fields of entertainment, education, marketing, and so forth and keeps expanding its territory. The features of digital storytelling: flexibility, universality, and interactivity can be enhanced by integration of multi-sensory digital media. The current sense convergence has limitation because of unbalance among each of sense technologies. Technologies of a sense of the olfactory and a sense of the taste are relatively insufficient. We proposed Sense cup as a device for digital storytelling and beverage based five senses convergence system as a solution.

\section{References}

1. Schmitt, B.H., Simonson, A.: Marketing Aesthetics: The Strategic Management of Branding, Identity and Image. Simon \& Schuster Inc. (1997)

2. Choi, H.-S.: Digital Storytelling. Communications of the Korea Information Science Society 21(2) (2003) (Korean)

3. Garzotto, F., Forfori, M.: Hyperstories and Social Interaction in 2D and 3D Edutainment Spaces for Children. In: HT 2006, August 22-25 (2006)

4. Gellersen, H.W., Beigl, M., Krull, H.: The MediaCup: Awareness Technology Embedded in and Everyday Object. In: Gellersen, H.-W. (ed.) HUC 1999. LNCS, vol. 1707. Springer, Heidelberg (1999)

5. Chung, H., Lee, C.-H.J., Selker, T.: Lover's Cups: Drinking Interfaces as New Communication Channels. In: CHI 2006 (April 2006)

6. Cho, H.S., Park, K.S., Hahn, M.: Tea Table Mediator: A Multimodal Ambient Display on the Table Exploiting Five Senses Convergence. In: Ma, L., Rauterberg, M., Nakatsu, R. (eds.) ICEC 2007. LNCS, vol. 4740, pp. 16-21. Springer, Heidelberg (2007)

7. McLuhan, M., Fiore, Q.: The Medium is the Message. The Penguin Press (1967)

8. Gaver, W.W., et al.: The Drift Table: Designing for Ludic Engagement. In: CHI 2004 (April 2004)

9. http://www.msl.ri.cmu.edu/projects/haptic_consortium

10. http://dstory.com/

11. http://www.temple.edu/ispr/examples/ex01_01_25a.html

12. http://www.storycenter.org

13. http://www.chiariglione.org/mpeg/working_documents/ explorations/RoSE/RoSE-Reqs . zip 